\title{
COMMENTARY
}

\section{PCR for the diagnosis of sepsis: hope or hype?}

\author{
Jacques Schrenzel* \\ See related research by Lehmann et al., http://ccforum.com/content/14/5/R186
}

\begin{abstract}
Molecular biology has not yet fully reached its ambitious goals in clinical bacteriology.

Notwithstanding the tremendous technical challenges, the detection of nucleic acids directly from the blood of septic patients has not been shown costeffective or even clinically relevant. Yet the potential for rapid molecular detection of circulating DNA (DNAemia) coupled to an educated antimicrobial drug adaptation has been repetitively advocated as a predicted breakthrough. Why do we still remain in such uncertainty?
\end{abstract}

In a previous issue of Critical Care, Lehmann and colleagues analyzed the potential impact of a commercially available PCR-based diagnostic method to test the whole blood of septic patients for bacterial DNAemia [1]. The evidence they collected is derived from their previous prospective observational trial showing reduction of inadequate treatment days [2] coupled with two other unrelated studies on outcomes [3,4]. The authors then developed a mathematical model to suggest that PCR-based detection of pathogens early during sepsis might reveal cost-effective, under-defined conditions. This is clearly a landmark paper in molecular microbial diagnostics but its predictions derive from numerous assumptions and therefore warrant a careful interpretation.

It is now obvious that adequate empiric therapy must be administered as soon as possible to septic patients in order to reduce mortality [5]. An early switch, however, from an empiric regimen initiated on clinical clues to an adapted therapy based on a probabilistic antimicrobial susceptibility, itself derived from the organism identification, might lead to more targeted and more appropriate therapy. This implies broadening the antimicrobial

\footnotetext{
*Correspondence: jacques.schrenzel@genomic.ch
}

Genomic Research and Bacteriology Laboratories, Service of Infectious Diseases, Geneva University Hospitals, Rue Gabrielle-Perret-Gentil 4, CH-1211 Geneva 14, Switzerland spectrum for an unexpected organism and/or narrowing the empiric coverage (for example, by stopping glycopeptides if an Escherichia coli strain is detected). Benefits might therefore derive from an improved clinical outcome and/or from savings due to drug adjustments. This strategy could also, however, lead to higher costs due to molecular diagnostics and adjusted therapy. And here comes the challenge to design such a healtheconomic study: one needs to bridge several medical disciplines (intensive medicine, laboratory medicine, epidemiology and infectious disease specialists, and so forth) to provide the full cost picture. This picture is needed to convince our hospital directors to implement costly but beneficial approaches, by showing them strong data supporting a paradigm shift in clinical microbiology.

Lehmann and colleagues have partially overcome these hurdles by compiling data from different studies [1]. Importantly, their approach makes several major assumptions: molecular testing does not provide false positive results (negative results are not considered here - hence, the issue of suboptimal detection sensitivity and the limited microbial diversity detection of the assay are not relevant here); the assay is performed only once, as soon as possible; results are rapidly delivered 24 hours per day to the physician in charge, who will immediately and optimally adjust therapy; the prevalence of positive blood cultures should be high (here more than 15\%, as observed in the emergency room wards and hence mostly detecting community-acquired infections); and, finally, there should be a high proportion of inadequate empirical treatments (here more than $25 \%$, which might be true mostly for hospital-acquired infections and therefore in wards other than the emergency room). The applicability of this approach is thus highly contextual as a function of the local epidemiology, of the respect of antimicrobial prescription guidelines, and assuming ideal 24 hours per day laboratory support. The cost-efficiency model should therefore be assessed by each potential user, for its intended target patient population and wards, by using its own numbers.

Finally, the compilation of data contains the risk that false positive PCR results (for example, the unexpected detection of DNA from Pseudomonas aeruginosa or yeasts/fungi) might overestimate the rate of inadequate 
antimicrobial therapy and inadequately support clinically-irrelevant, expensive and perhaps dangerous medical decisions. These limitations will have to be addressed in carefully designed studies.

In the future, such studies will have to confirm that the rapid availability of such PCR assays - a true logistical challenge to most diagnostic laboratories - can indeed be followed by changes in therapy and could prove costeffective, across different wards, hospitals and countries. Once validated, such molecular assays will probably be bundled with computer-assisted antimicrobial prescription and electronic reporting tools.

\section{Abbreviations}

$\mathrm{PCR}$, polymerase chain reaction.

\section{Competing interests}

The author declares that he has no competing interests.

Published: 26 January 2011

\section{References}

1. Lehmann LE, Herpichboehm B, Kost GJ, Kollef MH, Stüber F: Cost and mortality prediction using polymerase chain reaction pathogen detection in sepsis: evidence from three observational trials. Crit Care 2010, 14:R186.

2. Lehmann LE, Alvarez J, Hunfeld KP, Goglio A, Kost GJ, Louie RF, Raglio A, Regueiro BJ, Wissing H, Stuber F: Potential clinical utility of polymerase chain reaction in microbiological testing for sepsis. Crit Care Med 2009, 37:3085-3090.

3. Ibrahim EH, Sherman G, Ward S, Fraser VJ, Kollef MH: The influence of inadequate antimicrobial treatment of bloodstream infections on patient outcomes in the ICU setting. Chest 2000, 118:146-155

4. Kollef MH, Sherman G, Ward S, Fraser VJ: Inadequate antimicrobial treatment of infections: a risk factor for hospital mortality among critically ill patients. Chest 1999, 115:462-474

5. Kumar A, Ellis P, Arabi Y, Roberts D, Light B, Parrillo JE, Dodek P, Wood G, Kumar A, Simon D, Peters C, Ahsan M, Chateau D: Initiation of inappropriate antimicrobial therapy results in a fivefold reduction of survival in human septic shock. Chest 2009, 136:1237-1248.

doi:10.1186/cc9378

Cite this article as: Schrenzel J: PCR for the diagnosis of sepsis: hope or hype? Critical Care 2011, 15:111. 